Si I ver - act i vat ed r adi ophot ol um nescent gl ass: band assi gnment $s$ and a novel readout system usi ng a nodul at ed UV I aser di ode

\begin{tabular}{|c|c|}
\hline 著者 & Kur obor i Toshi o, Zheng Wang, Zhao C. \\
\hline $\begin{array}{l}\text { jour nal or } \\
\text { publ i cat i on titl e }\end{array}$ & $\begin{array}{l}\text { 11th Eur ophysi cal Conf er ence on Def ect s i n } \\
\text { I nsul at i ng Mat er i al s ( EUROD M 2010) }\end{array}$ \\
\hline page $r$ ange & $1-8$ \\
\hline year & 2010-01-01 \\
\hline URL & ht t p: //hdl . handl e. net /2297/25784 \\
\hline
\end{tabular}




\title{
Silver-activated radiophotoluminescent glass: band assignments and a novel readout system using a modulated UV laser diode
}

\author{
T Kurobori ${ }^{1}$, W Zheng ${ }^{1}$ and C Zhao ${ }^{1}$ \\ ${ }^{1}$ Graduate School of Natural Science and Technology, Kanazawa University, Kakuma, \\ Kanazawa 920-1192, Japan \\ E-mail: laser@kenroku.kanazawa-u.ac.jp (T. Kurobori)
}

\begin{abstract}
We investigated the assignments and characteristics of the X-ray-induced colour centres in a silver-activated radiophotoluminescent glass. The inducedabsorption spectrum was decomposed into six Gaussian bands, which were, in turn, attributed to the $\mathrm{Ag}^{+}, \mathrm{Ag}_{3}{ }^{+}$or $\mathrm{Ag}_{3}{ }^{2+}, \mathrm{Ag}_{2}{ }^{+}, \mathrm{Ag}^{2+}, \mathrm{Ag}^{0}$ and hole-trap centres, respectively, by means of optical and thermal measurements. All components of the blue and orange radiophotoluminescence (RPL) in a silver-activated phosphate glass were confirmed to be X-ray-induced colour centres. We also proposed and constructed a new readout system for the measurement of the RPL of the glass dosimeter that considers the characteristics of the radiation-induced bands.
\end{abstract}

\section{Introduction}

The silver-activated phosphate glass (designated PG:Ag) after the exposure to ionizing radiation emits an intense radiation-induced orange luminescence by the excitation with a pulsed ultraviolet (UV) laser, which is called radiophotoluminescence (RPL). Such a dose-dependent RPL in PG:Ag has long been examined [1-3] for large-scale use in personal and environmental monitoring of the dose. In particular, radiation-induced colour centres in PG:Ag have been extensively studied by means of optical spectroscopy $[1,3]$ and the electron spin resonance (ESR) method [2]. When PG:Ag is exposed to ionising radiation, various colour centres such as $\mathrm{Ag}^{0}, \mathrm{Ag}_{2}^{+}, \mathrm{Ag}^{2+}, \mathrm{Ag}_{3}{ }^{+}$and other silver ion species are produced and the existence of each these species has been confirmed by ESR studies [2]. However, the spectral contribution of these small clusters to the overall spectrum remains unknown.

As the basic characteristics of these $\mathrm{Ag}_{\mathrm{m}}{ }^{\mathrm{X}}{ }^{+}$centres have been studied, a readout system has been developed for the measurement of the RPL of the glass dosimeter [4-6] based on a time-resolved technique using a pulsed UV nitrogen laser excitation. The basic principle of the conventional reader is as follows [4]: the radiation-induced component (normally, the orange RPL with lifetime values of 2 $\mu \mathrm{s}<\mathrm{t} \leq 20 \mu \mathrm{s})$ is only integrated by subtracting the radiation independent short-term $(\mathrm{t} \leq 2 \mu \mathrm{s})$ and long-term $(\mathrm{t}>20 \mu \mathrm{s})$ components of the pre-dose fluorescence from the total RPL values. If these latter terms do not result from parasitic luminescence, then the time-resolved technique will not be necessary to discriminate the orange RPL from short- and long-term components. This will eliminate the instabilities of the pulsed laser power, the degradation of the induced colour centres and the complicated processing that is required due to the use of a pulsed $\mathrm{UV} \mathrm{N}_{2}$ laser excitation.

The purpose of this study is to further study the characteristics of the induced colour centres in PG:Ag after exposure to X-rays. In addition, a new reader for the measurement of the RPL of the glass 
dosimeter using a modulated continuous-wave (cw) UV laser diode and phase-sensitive method is proposed and constructed. Preliminary results using a home-made reader are also presented.

\section{Experimental Details}

A commercially available GD-450 dosimeter (AGC Techno Glass) was used as the radiophotoluminescent PG:Ag. Samples were cut from the original glass dosimeter plate to a size of approximately $10 \mathrm{~mm} \times 7 \mathrm{~mm} \times 1 \mathrm{~mm}$. The weight composition of the GD-450 dosimeter was $31.55 \%$ $\mathrm{P}, 51.16 \% \mathrm{O}, 6.12 \% \mathrm{Al}, 11.00 \% \mathrm{Na}$ and $0.17 \% \mathrm{Ag}$.

All samples were coloured by irradiation from an X-ray unit (energy: $8 \mathrm{keV}$ ) with a copper target operated at $30 \mathrm{kV}$ and $20 \mathrm{~mA}$. In this work, the samples were irradiated such that the absorbed doses ranged from $30 \mathrm{mGy}$ to $24.5 \mathrm{~Gy}$. Absorption, excitation and emission measurements were performed at room temperature using a Hitachi U-2010 UV-VIS and an F-2500 fluorescence spectrophotometer. Radiative lifetime measurements were performed using a time-resolved spectrofluorometer (Horiba, NAES-1100) with a resolution limit of sub-ns, which was operated based on the time-correlated multiphoton counting technique [7].

For the direct photo-induced reduction from $\mathrm{Ag}^{+}$ions to $\mathrm{Ag}^{0}$ centres in $\mathrm{PG}: \mathrm{Ag}$, this work used a regeneratively amplified 800-nm Ti:sapphire laser (Coherent, Mira and RegA) that emitted 80-fs, 250$\mathrm{kHz}$ mode-locked pulses. The femtosecond (fs) laser pulses were focused using a $20 \times$ objective lens $(\mathrm{NA}=0.40)$ to a depth of $250 \mu \mathrm{m}$ beneath the sample surface with the help of a computer-controlled 3D $X-Y-Z$ stage at a speed of 1000 and $50 \mu \mathrm{m} / \mathrm{s}$. The pulse energy ranged from 2.0 to $3.0 \mu \mathrm{J} /$ pulse and the spot diameter was approximately $2 \mu \mathrm{m}$.

\section{Results and Discussion}

\subsection{Optical characteristics and peak fitting analysis}

Figure 1(a) shows the absorption spectrum (solid line) of the X-ray-irradiated PG:Ag under an absorbed dose of $24.5 \mathrm{~Gy}$, and its spectrum was decomposed into the sum of separate Gaussian bands (indicated by a dotted line). The peak fitting analysis was carried out on the basis of the strong analogy with X-ray-irradiated silver-activated sodium chloride ( $\mathrm{NaC}: \mathrm{Ag}$ ) [8]. In the case of $\mathrm{NaCl}: \mathrm{Ag}$, the appropriate absorption band could be decomposed into six bands (not shown here) with peaks at 224, 276, 308, 335, 382 and $443 \mathrm{~nm}$, which are called the "A", "B", "C", "D", "E" and "F" bands, respectively. The peak positions of each band are in good agreement with those of the observed radiation-induced bands in $\mathrm{NaCl}: \mathrm{Ag}[9,10]$. The absorption peaks of the $\mathrm{RPL}$ in $\mathrm{NaCl}: \mathrm{Ag}$ are mainly attributed to bands "B", "C" and "D". The origin of these bands in $\mathrm{NaCl}: \mathrm{Ag}$ has already been clarified as follows: bands "B" and " $D$ " are related to $F$ (i.e., a single electron trapped at an anion vacancy) and $F_{2}$ (i.e., a pair of nearest-neighbour $F$ centres along a $<110>$ axis) centres with neighbouring $\mathrm{Ag}^{+}$, respectively, and the narrow "C" band is strongly related to the $\mathrm{Ag}^{0}$ centres located at large lattice defects. In the case of PG:Ag, the absorption bands could also be decomposed into six absorption bands from "A" to "F", with peaks at 225, 252, 270, 307, 354 and $424 \mathrm{~nm}$, respectively.

Figure 1 (b) shows the excitation spectra (Curves 1 and 2) and the corresponding emission spectra (Curves 3-5) after X-ray irradiation for PG:Ag. An absorption spectrum is also shown for comparison. The excitation spectra consist of two different spectra. One spectrum peaks at $308 \mathrm{~nm}$ (Curve 1) for emission at $560 \mathrm{~nm}$, and the other peaks at 270 and $345 \mathrm{~nm}$ (Curve 2) for emission at 450 and $560 \mathrm{~nm}$. The former corresponds to the decomposed 307-nm (corresponding to "D") Gaussian band, while the latter mainly corresponds to the decomposed 270- ("C") and 354-nm ("E") bands, respectively. The blue emission and some portion of the orange emission are strongly related to the $270-$ and $345-\mathrm{nm}$ excitation bands.

\subsection{Femtosecond laser exposure}

Recently, intense fs laser pulses with high-peak power densities $\left(>100 \mathrm{TW} / \mathrm{cm}^{2}\right)$ and high repetition rates $(>200 \mathrm{kHz})$ have enabled the direct precipitation of silver nanoparticles in silver-activated 
glasses without heat treatment [11]. In the case of silver-activated glasses, it is well known that irradiation with fs laser pulses as well as X-rays and subsequent annealing at high temperature $(\sim 770$ $\mathrm{K})$ for $30 \mathrm{~min}$ bring about the reduction of $\mathrm{Ag}^{+}$ions to $\mathrm{Ag}^{0}$ atoms and result in the formation of plasmonic nanoparticles, as observed using luminescence and ESR spectroscopy and transmission electron microscopy (TEM) [12].

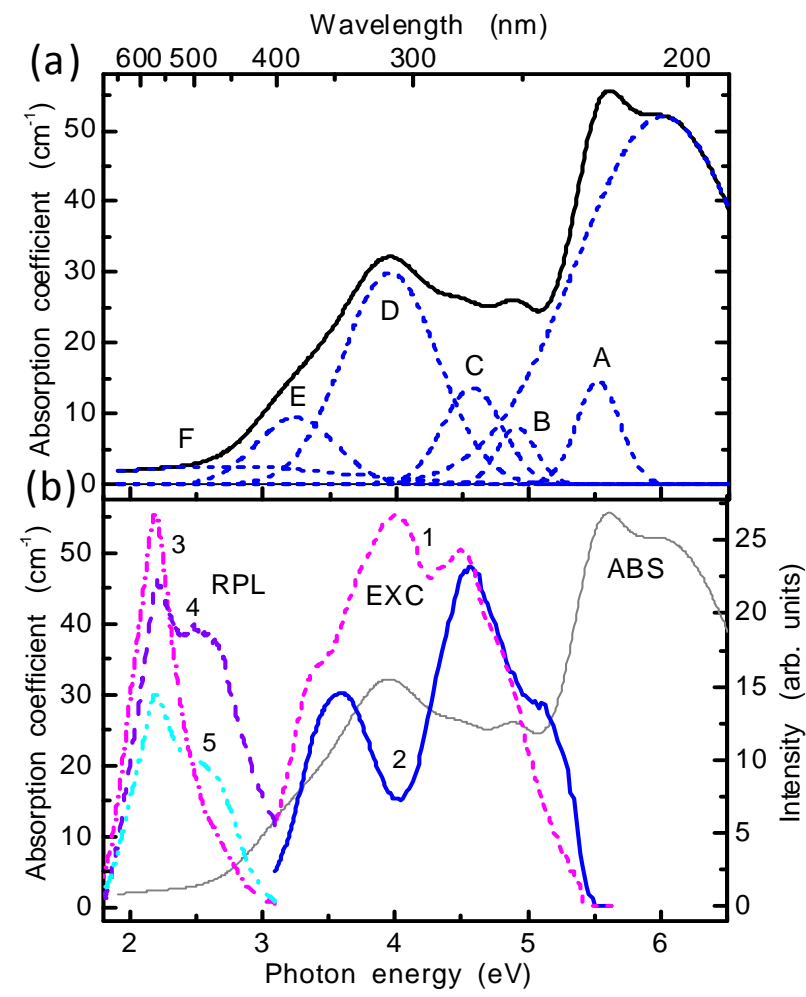

Figure 1. (a) Absorption and (b) excitation and RPL spectra of PG:Ag after X-ray irradiation with a dose of 24.5 Gy. The absorption spectrum was decomposed into the sum of separate Gaussian bands (dashed lines). Excitation was detected at $560 \mathrm{~nm}$ (Curve 1) and $450 \mathrm{~nm}$ (Curve 2) and RPL was excited at $308 \mathrm{~nm}$ (Curve 3), $270 \mathrm{~nm}$ (Curve 4) and 345 nm (Curve 5).

Figure 2 shows the absorption spectrum before (Curve 1) and after (Curves 2 and 3) irradiating fs laser pulses in multi-shot mode $\left(1.0 \times 10^{5}\right.$ shots in $\left.1 \mathrm{~s}\right)$ on the non-X-ray-irradiated PG:Ag. Note that Curves 2 and 3 show the difference in absorbance between the $800-\mathrm{nm}$ fs laser-irradiated and nonirradiated regions. The inset shows the excitation and emission spectra corresponding to the absorption spectrum for Curve 2. In the inset an excitation spectrum (solid line) for an emission at $560 \mathrm{~nm}$ (orange RPL) and emission spectra for excitations at $308 \mathrm{~nm}$ (dashed line) and at $345 \mathrm{~nm}$ (dasheddotted-line) are shown. These spectra of fs irradiation were in good agreement with those of the X-ray irradiation, as shown in Figure 1(b). For Curves 2 and 3, the pulse energy was $2.0 \mu \mathrm{J} /$ pulse and 3.0 $\mu \mathrm{J} /$ pulse at scanning rates of $1000 \mu \mathrm{m} / \mathrm{s}$ and $50 \mu \mathrm{m} / \mathrm{s}$, respectively. The spot diameter was approximately $2 \mu \mathrm{m}$. The light intensity of the laser beam irradiated on the sample was estimated to be $8.0 \times 10^{14} \mathrm{~W} / \mathrm{cm}^{2}$ for Curve 2 and $1.2 \times 10^{15} \mathrm{~W} / \mathrm{cm}^{2}$ for Curve 3. For Curve 2, a peak position at $315 \mathrm{~nm}$ of the absorption band was in good agreement with that of the X-ray irradiated absorption band, as shown in Figure 1(a).

On the other hand, for Curve 3 the difference in the absorption spectrum was decomposed into a sum of separate Lorentzian bands. As a result, the spectrum was dominated by an absorption band at $404 \mathrm{~nm}$ that could be ascribed to the surface plasmon resonance (SPR) of the formed silver nanoparticles [13]. Note that orange or blue emission for an excitation at $404 \mathrm{~nm}$ for Curve 3 could not be observed (not shown here). The other broad absorption band from 400 to $600 \mathrm{~nm}$ can be attributed to the hole-trap centres corresponding to the "F" band, as shown in Figure 1(a). 
The average diameter $\mathrm{d}$ of the embedded silver nanoparticles corresponding to Curve 3 was calculated from the decomposed absorption band using the formula $d=V_{f} \lambda_{p}{ }^{2} /(\pi c \Delta \lambda)$ [14], where $V_{f}$ is the Fermi velocity of the electrons in bulk silver $\left(\sim 1.39 \times 10^{6} \mathrm{~m} / \mathrm{s}\right), \Delta \lambda$ is the full-width at half maximum (FWHM) of the absorption band, and $\lambda_{\mathrm{p}}$ is the characteristic wavelength at which SPR occurs. The average size of the silver nanoparticles was calculated to be approximately $2.8 \mathrm{~nm}$, which is in a good agreement with the observation by TEM [12].

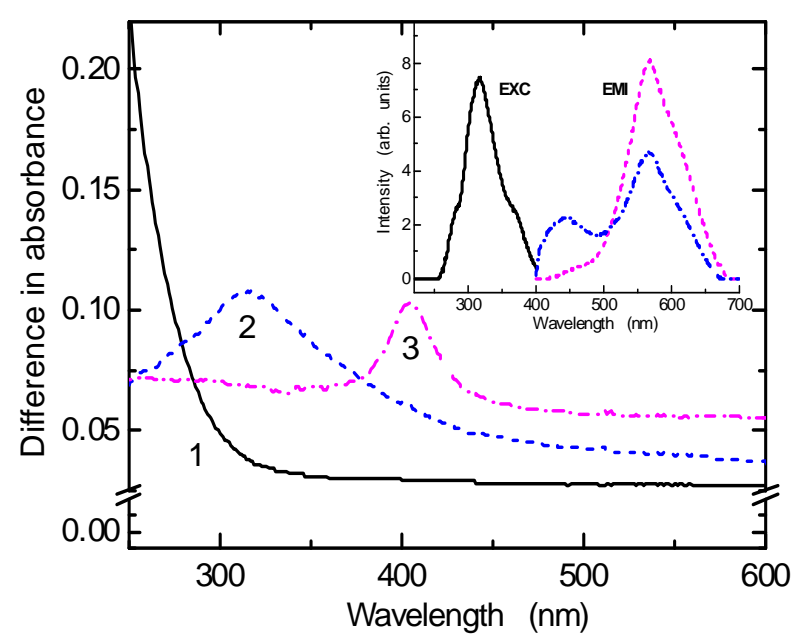

Figure 2. Absorption spectrum before (Curve 1) and after (Curves 2 and 3) irradiating fs laser pulses on the non-X-rayirradiated PG:Ag. The excitation (solid line) and emission (dashed and dashed-dottedlines) spectra corresponding to the absorption spectrum for Curve 2 are shown in the inset.

Note that irradiation with X-rays and fs laser pulses yields different absorption peak wavelengths of the $\mathrm{Ag}^{0}$ centres as described above: the former is $345 \mathrm{~nm}$, and the latter is $404 \mathrm{~nm}$. One of the reasons for this difference is that when fs laser pulses are focused inside the sample at a high repetition rate over $250 \mathrm{kHz}$, the temperature at the focal point increases to as high as several thousand $\mathrm{K}$ [15], a much higher temperature than that reached in normal heat treatment. As a result, highly successive fs laser pulses and a slow scanning rate caused cumulative heating around the focal point. Increasing the temperature greatly increased the average size of nanoparticles formed by fs laser irradiation, resulting in a red-shift of the peak wavelength. Moreover, Ma et al. [11] have reported that the light intensity on the order of $10^{15} \mathrm{~W} / \mathrm{cm}^{2}$ was high enough to generate multi-photon ionisation in the glass matrix, and the heat accumulated by the $250-\mathrm{kHz}$ fs laser resulted in the subsequent $\mathrm{Ag}$ nanoparticle growth process.

\subsection{Heat treatment}

Furthermore, to obtain additional evidence for the band assignments to $\mathrm{Ag}^{0}, \mathrm{Ag}_{2}{ }^{+}, \mathrm{Ag}^{2+}$ and $\mathrm{Ag}_{3}{ }^{+}$ centres, heat treatments were performed at temperatures from 295 (room temperature) to $523 \mathrm{~K}$. Before measuring the absorption, excitation and emission spectra of the RPL at each temperature after exposure to X-rays, a 30-min pre-heat was performed at $343 \mathrm{~K}$ to suppress the 'build-up' kinetics (i.e., the RPL centre concentration increases as a function of time after irradiation). Finally, the samples were thermally annealed at $673 \mathrm{~K}$ for $30 \mathrm{~min}$ to eliminate stable colour centres before they were used again.

Figure 3 shows absorption and excitation spectra at annealing temperatures of 295 and $523 \mathrm{~K}$. The band peaking at $345 \mathrm{~nm}$ due to the $\mathrm{Ag}^{0}$ centres shifted from $345 \mathrm{~nm}$ (solid line) at $295 \mathrm{~K}$ to $330 \mathrm{~nm}$ (dashed-and-double-dotted line) at $523 \mathrm{~K}$ with increasing annealing temperature. A similar phenomenon also appeared in optical absorption spectra taken on the gamma-irradiated, silver-doped silicate glass, where the absorption peak is blue-shifted from $345 \mathrm{~nm}$ (the characteristic wavelength of $\mathrm{Ag}^{0}$ atoms) at room temperature to $310 \mathrm{~nm}$ at $633 \mathrm{~K} \mathrm{[16].} \mathrm{A} \mathrm{blue} \mathrm{shift} \mathrm{from} 270$ to $260 \mathrm{~nm}$ was also 
observed for the $270 \mathrm{~nm}$ band with increasing annealing temperatures, as shown in figure 3(a). The blue shift can be explained by the reaction of neutral silver with $\mathrm{Ag}^{+}$, i.e., $\mathrm{Ag}^{0}+\mathrm{Ag}^{+} \rightarrow \mathrm{Ag}_{2}^{+}$, which therefore shifts the position of the $\mathrm{Ag}^{0}$ band. The $\mathrm{Ag}_{2}{ }^{+}$band with coupling to $\mathrm{Ag}^{0}$ atoms also blueshifts from the original position at room temperature. The complete set of results taken from 295 to $523 \mathrm{~K}$ showed that the maximum intensity of the blue excitation bands peaking at 270 and $345 \mathrm{~nm}$ occurred at $343 \mathrm{~K}$. These bands then decreased gradually in intensity with increasing temperature. Moreover, another larger band peaking at $244 \mathrm{~nm}$ (corresponding to "B") appeared after annealing at $523 \mathrm{~K}$, which may be attributed to the formation of $\mathrm{Ag}_{3}{ }^{+}\left(\mathrm{Ag}^{0}+\mathrm{Ag}_{2}{ }^{+} \rightarrow \mathrm{Ag}_{3}{ }^{+}\right) \mathrm{or} \mathrm{Ag}_{3}{ }^{2+}\left(\mathrm{Ag}^{+}+\mathrm{Ag}_{2}{ }^{+}\right.$ $\rightarrow \mathrm{Ag}_{3}{ }^{2+}$ ) from the diffusion and dimerisation of $\mathrm{Ag}_{2}{ }^{+}$ions.

In contrast, the band peaking at $308 \mathrm{~nm}$ due to $\mathrm{Ag}^{2+}$ without coupling to $\mathrm{Ag}^{0}$ centres remained in essentially the same peak position as shown in Figure 3(b); the intensity of the excitation band at 308 $\mathrm{nm}$ for the orange RPL at $560 \mathrm{~nm}$ increased monotonically with increasing temperature.

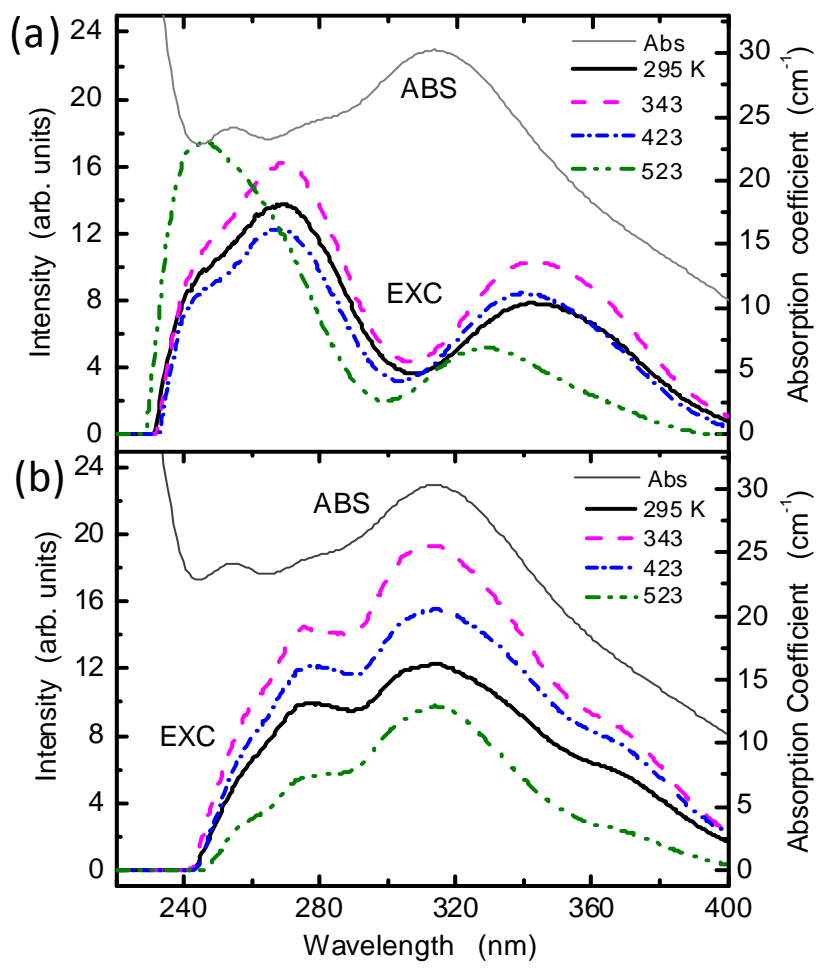

Figure 3. Excitation spectra of the irradiated PG:Ag at different annealing temperatures for detection at (a) $450 \mathrm{~nm}$ and (b) $560 \mathrm{~nm}$. Excitation spectra after different annealing temperatures: Excitation detected at $295 \mathrm{~K}$ (solid line), $343 \mathrm{~K}$ (dashed line), $423 \mathrm{~K}$ (dashed-and-dotted line) and $523 \mathrm{~K}$ (dashed-and-double-dotted line).

\subsection{RPL decay curve analysis}

Next, to investigate the change in the blue RPL over time by observing the 270 - and 345 -nm bands, the lifetime measurements were performed at room temperature. Table 1 summarises the results of the measured lifetimes at $450 \mathrm{~nm}$ for different doses, which were obtained by fitting the exponential components with the use of a least-squares iteration deconvolution method to the decay curves. The relative strength of the lifetimes are also given in parentheses in Table 1. The excitation wavelengths for the 270- and 345-nm bands were monochromatised using suitable interference filters. In the case of 345 -nm excitation, the lifetime values are almost independent of the absorbed doses ranging from 1.22 to $24.5 \mathrm{~Gy}$ and are about $5.6 \mathrm{~ns}$. In contrast, the lifetime values of $270-\mathrm{nm}$ excitation are strongly dependent on the dose, particularly for doses less than $2.45 \mathrm{~Gy}$, where the lifetime values drastically shorten to 2-3 ns. This result supports the other evidence on the different origin and structures of the 270- and 345-nm bands. In the case of lower doses, photoluminescence (PL) at $302 \mathrm{~nm}$ excited by the $\mathrm{Ag}^{+}$band becomes predominant, and thus a shoulder part of the PL completely overlaps a blue RPL at 
$450 \mathrm{~nm}$. If the PL is detected instead of emission due to the $\mathrm{Ag}_{2}{ }^{+}$centres, the lifetime value becomes much longer, about $8600 \mathrm{~ns}$, as already reported in [8]. Therefore, one reason for these shorter lifetime values may be the 'perturbation effect' of the excited state $\left(\mathrm{Ag}_{2}{ }^{+}\right)^{*}$ level by stronger emission of PL. However, more detailed information is needed for a satisfactory explanation.

Table 1. Radiative lifetime values as measured for the blue RPL at $450 \mathrm{~nm}$ excited at $270 \mathrm{~nm}$ and $345 \mathrm{~nm}$ for PG:Ag. Xray doses from 1.22 to $24.5 \mathrm{~Gy}$.

\begin{tabular}{ccc}
\hline Doses (Gy) & $\begin{array}{r}\text { Lifetime (ns) } \\
\text { @270 nm }\end{array}$ & $\begin{array}{r}\text { Lifetime (ns) } \\
\text { @345 nm }\end{array}$ \\
\hline 1.22 & $3.31(79.5 \%)$ & $5.56(71.7 \%)$ \\
\hline 2.45 & $2.40(55.7 \%)$ & $5.44(76.8 \%)$ \\
\hline 7.35 & $6.32(63.3 \%)$ & $5.67(73.0 \%)$ \\
\hline 12.2 & $6.38(62.0 \%)$ & $5.71(72.6 \%)$ \\
\hline 24.5 & $6.15(63.8 \%)$ & $5.63(75.7 \%)$ \\
\hline
\end{tabular}

\subsection{RPL readout system}

As previously described, the blue emission at $450 \mathrm{~nm}$ and orange emission at $560 \mathrm{~nm}$ in PG:Ag were confirmed to be the radiation-induced colour centres. Therefore, it is not necessary to use a timeresolved technique to discriminate the orange RPL from short- and long-term components, which were regarded as parasitic luminescence. We have proposed and constructed a new readout system with a modulated cw UV laser diode as an excitation and phase-sensitive method instead of a time-resolved technique.

Figure 4 presents a schematic view of the experimental setup for the RPL measurement. The excitation source is a $16-\mathrm{mW}$, cw laser diode (Coherent, CUBE $375-16 \mathrm{C}$ ) at $37^{\prime} 1 \mathrm{~nm}$ that provides a linearly polarised output beam with $\mathrm{M}^{2}<1.2$. The laser diode was modulated by a rectangular waveform at frequencies up to $70 \mathrm{kHz}$ (average power: $\mathrm{P}_{\text {ave }}=2.5 \mathrm{~mW}$, peak power density: $\mathrm{P}_{\text {peak }}<50$ $\mathrm{W} / \mathrm{cm}^{2}$ ), and a portion of the driving voltage was fed as a reference signal of a lock-in amplifier (NF, LI-574A). The blue and orange RPLs were detected with a photomultiplier (Hamamatsu, R1463) through appropriate filters and then amplified by a lock-in-amplifier.

Figure 5 shows typical orange RPL output in the X-ray irradiated PG:Ag (a) with various doses from 82 to $320 \mathrm{mGy}$ and (b) with a dose of $30 \mathrm{mGy}$. In general, the glass dosimeter GD-450 used in this work is set in a plastic holder with embedded metal and plastic filters used for the discrimination of radiation [6]. In Figure 5(a), X-ray irradiation was determined without the plastic holders, while in Figure 5(b) it was measured with plastic holders partially shielded by metal and plastic filters. The measurements were performed according to the following procedure: first, after X-ray irradiation, the background signal (dashed line) corresponding to the pre-dose fluorescence of the glass surface, stray light and dirt was obtained from the samples in non-irradiated areas. Second, the orange RPL signal was measured by repeatedly scanning irradiated areas of the samples. Finally, the RPL intensities were obtained by subtracting the averaged background signal from the averaged RPL value. Figure 5(a) shows experimental results of the relationship between the RPL intensity and the absorbed doses of the glass dosimeter, demonstrating a linear relationship. Figure 5 (b) shows the measured RPL intensity obtained from the X-ray irradiated PG:Ag with a dose of $30 \mathrm{mGy}$ with plastic holders containing a sample partly shielded by metal and plastic filters. In general, the use of various filters 
gives additional information about incident radiation qualities such as gamma-, beta- and X-rays by measuring the RPL intensity ratio [17]. In this case, the ratio of X-rays was similar to that of beta-rays, which may be due to the low energy photons of about $8 \mathrm{keV}$. Consequently, the new readout system with a modulated cw UV laser diode as an excitation and phase-sensitive method has considerable potential for the measurement of the RPL of glass dosimeters.
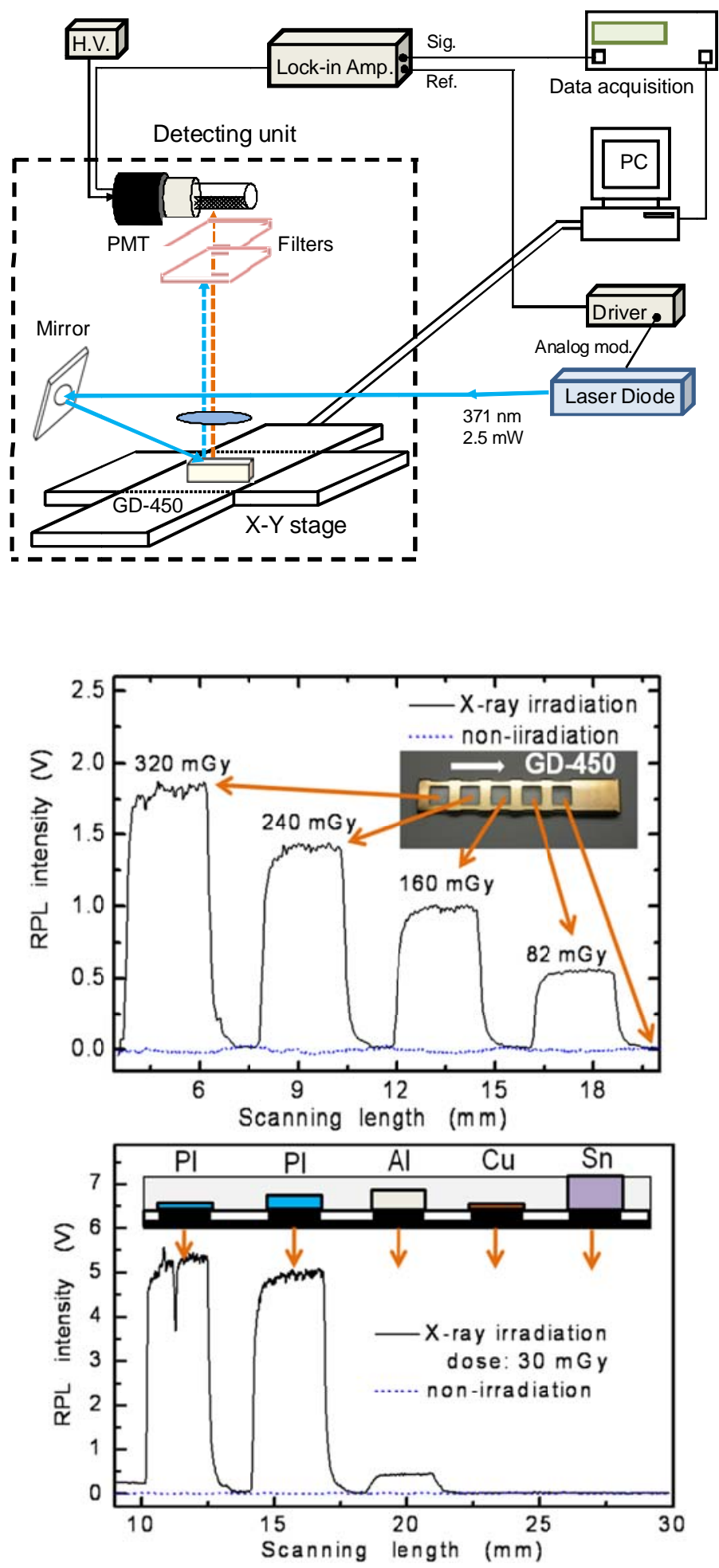

Figure 4. Schematic setup for the RPL measurement of the glass dosimeter.

Figure 5. Measured RPL intensity as a function of the horizontal scanning length (a) without the plastic holders and (b) with the plastic holders containing metals and plastic filters. The filters and thicknesses (in $\mathrm{mm}$ ) were: $\mathrm{Pl}(0.22), \mathrm{Pl}$ (0.5), $\mathrm{Al}$ (0.7), $\mathrm{Cu}$ (0.2) and $\mathrm{Sn}$ (1.2). 'Pl' indicates a plastic filter. The PG:Ag sample was irradiated with X-rays (a) with doses ranging from 82 to $320 \mathrm{mGy}$ and (b) with a dose of $30 \mathrm{mGy}$. 


\section{Conclusion}

The data obtained in this study allow for the following conclusions:

(1) An absorption band of X-ray irradiated PG:Ag could be decomposed into six Gaussian bands, marked as "A" to "F", peaking at 225, 252, 270, 307, 354 and $424 \mathrm{~nm}$ for PG:Ag, respectively.

(2) Through fs laser pulse exposure, heat treatment, and lifetime measurements, the "A" band at 225 $\mathrm{nm}$, the "B" band at $252 \mathrm{~nm}$, the "C" band at $270 \mathrm{~nm}$, the "D" band at $307 \mathrm{~nm}$, the "E" band at $354 \mathrm{~nm}$, and the "F" band at $424 \mathrm{~nm}$ were attributed to $\mathrm{Ag}^{+}, \mathrm{Ag}_{3}{ }^{+}$or $\mathrm{Ag}_{3}{ }^{2+}, \mathrm{Ag}_{2}{ }^{+}, \mathrm{Ag}^{2+}, \mathrm{Ag}^{0}$ and hole-trap centres, respectively.

(3) In the absorbed-dose range of 1.22-24.5 Gy, no components of the blue and orange emissions could be attributed to dirt or any pre-dose. All components of the blue and orange emissions in PG:Ag were confirmed to be radiation-induced colour centres. In particular, the lifetime of the blue RPL excited at $345 \mathrm{~nm}$ remained constant for various doses, while that of the blue RPL excited at $270 \mathrm{~nm}$ centres were strongly dependent on the absorbed doses.

(4) Preliminary experimental results were demonstrated using a home-made reader based on a modulated UV laser diode and a phase-sensitive technique that we proposed and constructed.

\section{Acknowledgements}

We would like to thank Ms. Y. Miyamoto and Dr. Yamamoto at Chiyoda Technol Corporation for their contributions to the sample preparation and Prof. H. Nanto at Kanazawa Institute of Technology for his valuable discussions. We would also like to thank Dr. M. Sakakura, Dr. Y. Shimotsuma, Prof. K. Miura and Prof. K. Hirao at Kyoto University for their contributions to the femtosecond laser experiments.

\section{References}

[1] Schulman J H, Ginther R J, Klick C C, Alger R S and Levy R A 1951 J. Appl. Phys. 221479

[2] Yokota R and Imagawa H 1966 J. Phys. Soc. Japan 231038

[3] Feldmann T and Treinin A 1967 J. Chem. Phys. 472754

[4] Schneckenburger H, Regulla D F and Unsöld E 1981 Appl. Phys. A 2623

[5] Piesch E, Burgkhardt B, Fischer M, Röber H G and Ugi S 1986 Rad. Prot. Dos. 17293

[6] Juto N 2002 FB News No.308 10-14 (in Japanese)

[7] Kurobori T, Kanasaki T, Imai Y and Takeuchi N 1988 J. Phys. C Solid State Phys. 21 L397

[8] Kurobori T, Zheng W, Miyamoto Y, Nanto H and Yamamoto T 2010 Opt. Mater. 321231

[9] Shulman J H and Compton W D 1963 Color Centers in Solids (New York: Pergamon) p 160

[10] Burstein E, Oberly J J, Henvis B and White M 1955 Phys. Rev. 86255

[11] Ma N H, Ma H L, Zhong, M J, Yang J Y, Dai Y, Ye G, Yue Z Y, Ma G H and Qiu J R 2009 Mater. Lett. 63151

[12] Shimotsuma Y, Hirao K, Kazansky P G and Qiu J 2005 Japan. J. Appl. Phys. 444735

[13] Tanahashi I, Yoshida M, Manabe Y, Tohda T 1995 J. Mater. Res. 16362

[14] Mie G 1908 Ann. Phys. 25377

[15] Sakakura M, Shimizu M, Shimotsuma Y, Miura K and Hirao K 2008 Appl. Phys. Lett. 93 231112

[16] Espiau de Lamaestre R, Béa H, Bernas H, Belloni J and Marignier J L 2007 Phys. Rev. B 76 205431

[17] Juto N 2002 FB News No.309 5-9 (in Japanese) 